\title{
Resilienz in klinischen Populationen
}

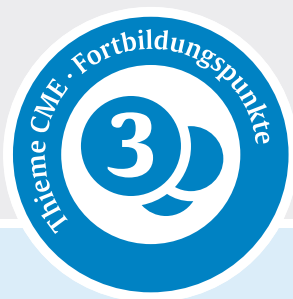

Christina Berndt, Andrea Pfennig

Klinik für Psychiatrie und Psychotherapie, Carl Gustav Carus Universitätsklinikum, Technische Universität Dresden

\section{ZUSAMMENFASSUNG}

Resilienz beschreibt den Prozess der Aufrechterhaltung und/ oder kurzfristigen Wiederherstellung von Gesundheit nach Exposition mit einem Stressor. Resilienzforschende sind mit einer Vielzahl von Definitionen, Operationalisierungen und Messverfahren in Resilienzstudien konfrontiert. Die Untersuchung von Resilienzfaktoren und -mechanismen in klinischen Populationen birgt methodische Herausforderungen, verspricht jedoch, das Verständnis für die Entwicklung, Manifestation und den Verlauf psychischer Störungen zu vertiefen.
Die Autorinnen diskutieren konzeptuelle Überlegungen und deren Implikationen für die Resilienzforschung in klinischen Populationen. Hier liegt der Fokus auf der Erfassung von Gesundheitsmarkern bereits vor Auftreten (spezifischer) Stressoren, um konzeptuelle Verlaufshypothesen in klinischen Populationen zu prüfen. In Zukunft sind prospektive, longitudinale Studien notwendig, welche die dynamischen und prozessualen Veränderungen psychischer Gesundheit in Reaktionen auf evidente Risikofaktoren und Stressoren abbilden und mittels innovativer Analysemethoden untersuchen. Empirisch gewonnene Ansätze für Prävention und Intervention werden die Erhaltung von Lebensqualität, Wohlbefinden, psychosozialem und beruflichem Funktionsvermögen angesichts psychischer Stressoren fokussieren.

\section{Konzeptuelle Herausforderungen der Resilienzforschung}

Vor über 60 Jahren beobachteten Entwicklungspsychologen, dass sich Kinder und Jugendliche trotz chronischer widriger Umstände wie Armut, Bürgerkriegen, Naturkatastrophen und familiärer Konflikte wesentlich häufiger als erwartet zu gesunden und erfolgreichen Erwachsenen entwickelten [1]. Diese inzwischen wohldokumentierte Beobachtung, dass einige Menschen keine oder nur geringe stressbezogene längerfristige Einschränkungen erleben, obwohl sie vergleichbaren Widrigkeiten und Stressoren in ihrem Leben ausgesetzt sind, führte zu den heutigen Kernfragen der Resilienzforschung [2, 3]. Diese Fragen fallen auf fruchtbaren Boden - das Interesse an Resilienz wächst im Kontext des fortlaufenden Paradigmenwechsels von krankheits- zu gesundheitsorientierter Forschung und Versorgung [4]. Die neue Perspektive richtet den Blick von der Korrektur und Vermeidung pathologischer Prozesse auf die Ursprünge von Gesundheit und die Förderung protektiver Mechanismen [5].

Es existieren eine Vielzahl von Entwicklungsverläufen in Reaktion auf Widrigkeiten und Stressoren, die durch interagierende genetische, biologische, psychologische, interpersonelle und umgebungsbedingte Variablen beeinflusst werden [2, 3, 6]. > Abb. 1 illustriert die am häufigsten beschriebenen Verläufe psychischer Gesundheit und Funktionsfähigkeit nach Auftreten schwerwiegender Stressoren. Wir erkennen Anpassungsprofile mit a. keinen oder lediglich milden und stabilen Symptomen,

b. minimalen Einschränkungen und vorrübergehenden milden bis mittelgradigen Symptomen, die nach kürzester Zeit nachlassen,

c. gradueller Abnahme mindestens mittelgradiger Symptome bis zu einem gesunden Funktionsniveau,

d. verzögerter, gradueller Zunahme von Symptomen und Funktionseinschränkungen sowie

e. Symptome und Einschränkungen, die stabil über lange Zeit nach Stressorexposition bestehen bleiben $[3,7,8]$.

3 der beobachteten Anpassungsprofile in Reaktion auf eine schwerwiegende Widrigkeit führen im Verlauf zu einem Zustand psychischer Gesundheit und Funktionsfähigkeit ( $a$, b und c) und können aufgrund dessen als resiliente Verläufe bezeichnet werden. Sie unterscheiden sich hinsichtlich des Ausmaßes der ursprünglichen Stressreaktion und, im Falle von b und c, in der Zeit bis ein Zustand von Gesundheit erreicht ist [7]. Es wird angenommen, dass die zugrunde liegenden Wirkprozesse dieser 3 Profile divergieren, d. h. Wirkfaktoren und -prozesse unterscheiden sich zwischen Menschen, die weder Symptome noch Funktionseinbußen erleben von jenen, deren ursprüngliche Symptome und Einschränkungen schnell sistieren oder aber von jenen, deren Symptome und Einschränkungen allmählich abnehmen. Für diese potenziell unterschiedlichen Wirkfaktoren ist zu prüfen, durch welche Interventionsstrategien zu welchen Zeitpunkten sie am effektivsten 


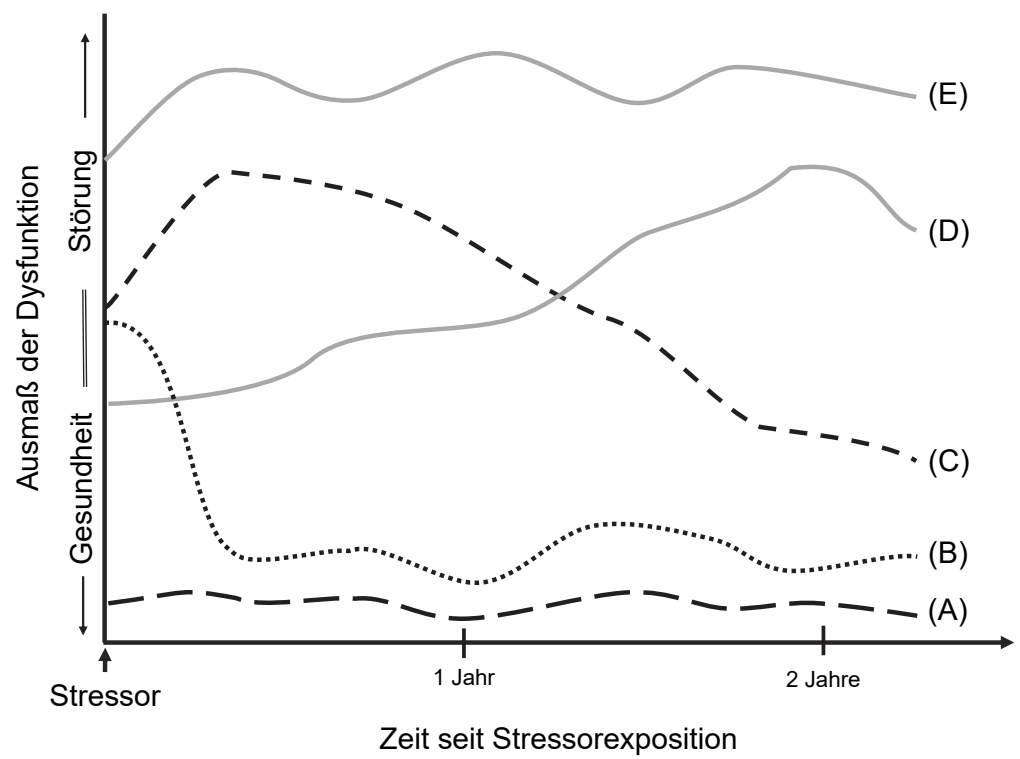

- Abb. 1 Schematische Darstellung gängiger Verläufe psychischer Gesundheit und Funktionsfähigkeit in Reaktion auf einen schwerwiegenden Stressor (nach Daten aus [7]).

beeinflusst werden können [7, 8]. Aus diesem Grunde kann die weitere Unterteilung resilienter Verläufe in

- Resistenz,

- Resilienz im engeren Sinne und

- Genesung („recovery“) funktional sein.

Über die eindeutige und vor allem evidenzbasierte Differenzierung beobachtbarer Gesundheitsverläufe und deren vermutlich zugrunde liegenden Wirkprozessen besteht jedoch noch Uneinigkeit, sodass die vorgestellten Ideen vorerst zu prüfende Hypothesen bleiben [8]. Den gängigen Definitionsversuchen von Resilienz ist gemein, dass sie einen „Prozess der effektiven Anpassung an stressreiche oder potenziell traumatische Ereignisse“ beschreiben [4]; eine Definition, die alle zuvor beschriebenen Verläufe (Resistenz, Resilienz im engeren Sinne und Genesung) einschließt. Aus diesem Grund finden wir zahlreiche und sehr verschiedene Ansätze zur Operationalisierung und Messung von Resilienz, was den Vergleich von Studienergebnissen erheblich erschwert [4, 9].

- Abb. 1 illustriert mögliche Gesundheitsverläufe nach Stressorexposition, wie sie bspw. in einer populationsbasierten Stichprobe nach einer Naturkatastrophe beobachtbar sein können [7]. Diese Daten enthalten keine Informationen über das Funktionsniveau vor der Konfrontation mit dem Stressor. Wir können jedoch davon ausgehen, dass diese populationsbasierten Stichproben Personen mit diversen, bereits vor dem Auftreten des Stressors, bestehenden Störungen einschließen - über deren Gesundheitsverläufe jedoch keine gesonderten Daten vorliegen. Es gibt keinen Grund zur Annahme, dass Resilienzprozesse und -mechanismen enden, sobald eine Störung diagnostiziert wird [10]. Die illustrierten Daten erhalten zudem keine Informationen über die Stressorbelastung im Verlauf nach dem initialen Stressor (hier eine Naturkatastrophe), obwohl diese, gemäß dem aktiven, dynamischen und prozessualen Charakter des Konzepts, die weitere Entwicklung der psychischen Gesundheit und Funktionsfähigkeit maßgeblich beeinflussen können. Die Resilienzforschung in klinischen Populationen birgt die Möglichkeit, zusätzliche Erkenntnisse über Wirkmechanismen und potenzielle Interventionsstragien und -zeitpunkte über Gesundheitsverläufe hinweg zu gewinnen.

\section{Methodische Herausforderungen der Resilienzforschung}

Die Definition von Resilienz als dynamischer Prozess in Reaktion auf einen Stressor birgt klare Konsequenzen für Studiendesign und Operationalisierung der Variablen. Das einfachste Studiendesign zur Untersuchung von Resilienz erfasst relevante Risiken/Stressoren/Widrigkeiten und die Adaption über eine gewisse Zeitspanne $[4,6,11]$ durch wiederholte Messung von Ergebnisvariablen vor und nach Stressorexposition. Je mehr Messzeitpunkte Stressorlast und Resilienzmarker erfassen, desto detaillierter und realistischer kann die Dynamik von Entwicklungsverläufen abgebildet werden [12].

\section{Untersuchung von Widrigkeiten und Stressoren in der Resilienzforschung}

Resilienz kann nicht ohne Kenntniss über relevante Stressoren oder Widrigkeiten beobachtet und untersucht werden. Die Ereignisse müssen per Definition mit einem signifikanten Risiko einhergehen und unter typischen Bedingungen 
bei der Mehrheit der Betroffenen Anpassungsprozesse herausfordern. Alle Arten von Stressoren (akut und chronisch, psychologisch, biologisch, sozial und ökonomisch) sollen möglichst detailliert erfasst werden (Anzahl, Schweregrad, Dauer und assoziierte Wirkfaktoren) [11]. Dabei treffen wir auf eine unüberschaubare Zahl verschiedener Stressoren, die sich in ihrer Auswirkung auf einzelne Menschen und deren spezifische Umwelt gravierend unterscheiden können, sodass Erkenntnisse lediglich in ihrem spezifischen Kontext interpretiert werden können [4, 6]. Kalisch et al. [12] schlagen aus diesem Grund die Erfassung einer Vielzahl möglicher Stressoren und deren Auswirkung im Rahmen einer dimensionalen Stressorbelastung vor. Dieses Vorgehen kann nach dem Gesetz der großen Zahl individuelle Unterschiede ausmitteln und Interpretationen über spezifische Kontexte hinweg erlauben.

\section{Resilienz beobachten und messen}

Die Wahl eines Messverfahrens und geeigneter Variablen hängt unumgänglich von der angewandten Definition von Resilienz ab. Ein positiver Verlauf mit langfristig keinen oder milden Symptomen und Funktionseinschränkungen kann wie besprochen auf unterschiedlichen Wegen erreicht werden (Resistenz, Resilienz im engeren Sinne und Genesung). In der Vergangenheit wurden diese 3 Profile oft in einer Gruppe als „resiliente“ Personen, die nicht die Kriterien einer psychischen Störung erfüllten, zusammengefasst und den „nicht resilienten“, klinisch erkrankten Personen entgegengesetzt. In diesem binären Denkmuster (gesund versus krank) sagen jene Faktoren Gesundheit vorher, die auch Krankheit vorhersagen - nur die Richtung der Vorhersage ändert sich. Das heißt, wenn geringe soziale Unterstützung die Wahrscheinlichkeit für die Entwicklung einer Störung erhöht, dann wird eine ausgeprägte soziale Unterstützung das Ausbleiben der Entwicklung einer Störung vorhersagen. In diesem Denkmuster werden Indikatoren für Ressourcen und Gesundheit einen zu geringen Mehrwert zur Risikoforschung leisten [4]. Damit Resilienz und Risiko nicht als gegensätzliche Pole eines Kontinuums, sondern als 2 verschiedene Faktoren behandelt werden, braucht es facettenreiche Konzepte im Verständnis von Gesundheit [13].

Resilienzmaße sollten den Erhalt bzw. die Wiederherstellung der „normalen“ Entwicklung und Funktionsniveaus (z. B. Erleben positiver Emotionen, Wohlbefinden, Nachgehen zielorientierter und persönlich bedeutsamer Aktivitäten) über eine Vielzahl von Bereichen und über eine umfassende Zeitperiode abbilden [4, 8, 11, 13]. Die Ergänzung klassischer Morbiditätsparameter wie Anzahl und Schweregrad von Symptomen und Episoden durch gesundheitsbezogene Variablen wird auch durch Studien zu patientenorientierter Therapiezielforschung gestützt. So bewerteten bspw. Patienten mit bipolarer Störung Therapieziele, die Störungsbewältigung, Lebensqualität, Beteiligung am therapeutischen Geschehen und Nebenwirkungen von Medikamente betreffen als bedeutsamere
Zieldimensionen als jene, die genannte Morbiditätsparameter betreffen [14].

In den vergangenen Jahren wurde eine Vielzahl von Selbstbeurteilungsfragebögen zur Berechnung von Resilienzwerten entwickelt, die vorrangig Ressourcen und protektive Faktoren erfragen und deren empirische Validierung in prospektiven Studien als möglicher Prädiktor für eine resiliente Entwicklung noch aussteht [15]. Gerade im Kontext klinischer Populationen wird die Beobachtung von Symptomen und Dysfunktionen jedoch von unbestreitbaren Interesse bleiben. Die Erhebung diskreter, kontinuierlicher Werte zur Abbildung der Symptomausprägung bietet mehr Informationen als der traditionelle binäre Ansatz, der lediglich die Kategorien krank und gesund gegenüberstellt. Die Erfassung der Symptomausprägung zu mehreren Zeitpunkten vor, während und nach Stressorexposition ermöglicht aufgrund der umfangreichen Datenbasis die Anwendung komplexer analytischer Methoden, um dem dynamischen, interaktiven und prozessualen Charakter von Resilienz gerecht zu werden.

\section{Modellieren komplexer, dynamischer Prozesse in klinischen Populationen}

Resiliente Entwicklung kann idealerweise durch Resilienzfaktoren vorhergesagt werden. Diese empirisch fundierten Faktoren, zumeist stabile Charakteristika und Prädispositionen, erhöhen die Wahrscheinlichkeit für Resilienz nach Exposition mit einem Stressor, ebenso wie Vulnerabilitätsfaktoren diese Wahrscheinlichkeit verringern. Potenzielle Resilienzfaktoren werden auf 3 Funktionsebenen untersucht:

- auf individueller Ebene (z. B. psychologisch,

biologisch, neurobiologisch),

- auf sozialer Ebene (z. B. emotional-responsiver Erziehungsstil, soziale Zugehörigkeit und Freundschaften, unterstützende und liebevolle partnerschaftliche Beziehungen) und

- auf gesellschaftlicher Ebene (z. B. institutionelle und ökonomische Faktoren, Hilfesysteme wie Präventions- und Beratungsangebote) $[4,11]$.

Resilienzfaktoren (wie adäquate Emotionsregulationsfähigkeit) wirken, so die Idee, durch die Aktivierung von Resilienzmechanismen (bspw. Regulation aufsteigender Wut in einer frustrierenden Arbeitssituation durch Atemtechniken und positive Selbstverbalisation). Faktoren und Mechanismen können sich im Rahmen von Resilienzprozessen im Laufe der Zeit durch Erfahrung und Lernen, z. B. Entwicklung neuer Emotionsregulationstechniken, weiterentwickeln und so die Wahrscheinlichkeit für Resilienz erhöhen $[6,12]$.

Resilienzfaktoren können direkten Einfluss auf das Ergebnis nehmen oder indirekt die Effekte des Stressors moderieren. Das führt zu komplexen Interaktionen zwischen Stressoren, protektiven Faktoren und Outcomes unter Einbe- 
zug der Entwicklung durch Erfahrung [3, 4]. Die Modellierung einer psychischen Störung als Netzwerk bietet eine innovative Möglichkeit, sich der Komplexität des Resilienzphänomens insbesondere in klinischen Populationen anzunähern. In diesem Verständnis werden Störungen als sich kausal bedingende Netzwerke von Symptomen modelliert, in denen die Aktivierung eines Symptoms (Knotenpunkt) zur Aktivierung verknüpfter Symptome (weitere Knotenpunkte) führt. In diesem Sinne wäre das Auftreten von Symptomen kausal verknüpft: Schlafstörungen führen bspw. zu Müdigkeit, führen zu Konzentrationsstörungen, führen zu Funktionseinschränkungen, führen zu Selbstwertreduktion. Die Aktivierungsstärke des Netzwerkes ist abhängig von den Eigenschaften der Verbindungen und wird durch biologische, psychologische und soziale Mechanismen realisiert. Die Aktivierung einzelner Symptome durch externe Stressoren führt in Netzwerken mit hoher Verbindungskraft dazu, dass alle Knotenpunkte des Netzes hoch aktiviert sind - eine Störung oder Episode wird manifest. Diese entscheidenden Wendepunkte im Übergang von Gesundheit zu Krankheit („tipping points“) spielen auch in der Untersuchung von Resilienzfaktoren eine besondere Rolle [16, 17].

Genesung bedeutet, dass Symptome deaktiviert werden und/oder die Verbindung zwischen Symptomen sich auflöst. Resiliente Netze geraten trotz substanzieller aktivierender Faktoren aufgrund ihrer geringeren Konnektivität der Symptome (Knotenpunkte) nicht in den Zustand genereller hoher Aktivierung [17, 18]. Dieses Phänomen beschrieb van Borkulo et al. [19] in einer Studie depressiver Patienten: Probanden mit Netzwerken geringerer Konnektivität waren nach 2 Jahren deutlich häufiger genesen als jene mit hoher Interkorrelation zur initialen Erhebung. Die Forschenden konnten in diesem Zusammenhang die besondere Rolle der Symptome Schulderleben, Müdigkeit und Energielosigkeit für die Persistenz der Störung über einen Zeitraum von 2 Jahren aufzeigen. Diese Form der Analyse wurde in Studien bereits u. a. bei posttraumatischer Belastungsstörung, Trauerreaktionen, Zwangsstörungen, Schizophrenie, sozialen Angststörungen und Substanzkonsumstörungen angewandt [17]. Im Rahmen von hybriden Symptom-und-Resilienzfaktoren Netzwerken (HSR) schlagen Kalisch und Kollegen vor [18], Resilienzfaktoren als zusätzliche Knotenpunkte zu den Symptomen in Netzwerke zu integrieren und deren Konnektivität zu einzelnen Symptomen und Bedeutung für die Aktivierungsstärke des gesamten Netzes zu prüfen. Bisherige Netzwerkanalysen basieren vorrangig auf querschnittlich erhobenen, korrelativen Daten, die naturgemäß keine Aussagen zur Kausalität ermöglichen. Aus diesem Grund, und um dem prozessualen Charakter des Konzepts Resilienz gerecht zu werden, braucht es dringend prospektive longitudinale Studien [17].

\section{Ausblick}

Resilienz beschreibt den Prozess der Aufrechterhaltung und/oder kurzfristigen Wiederherstellung von Gesundheit nach Exposition mit einem Stressor. Resilienz ist nicht „in“ einer Person, sondern entwickelt sich aus einem interaktiven Prozess, der über viele Funktionsebenen wirkt - zellulär, individuell, sozial und gesellschaftlich [20].

Das Verständnis von Resilienz als aktiver, dynamischer Prozess der Adaptation birgt Herausforderungen für Operationalisierung, Studiendesign und Analysemethoden, die weiter diskutiert, erprobt und verfeinert werden müssen. In klinischen Populationen liegt der Fokus zunächst auf der Charakterisierung der Anpassungsprozesse, die vor, während und nach Stressorbelastung mit evidenten Risiken und Stressoren deutlich werden, um die bestehenden konzeptuellen Überlegungen zu prüfen. Der Wahl und Erprobung von Resilienzmaßen kommt dabei besondere Bedeutung zu, um die Perspektive um patientenorientierte Werte außerhalb der binären Beurteilungsperspektive krank-versus-gesund zu erweitern. Die Abbildung des komplexen, dynamischen und interaktiven Prozesses über eine lange Zeitspanne kann mittels innovativer Methoden wie Netzwerkanalysen gelingen.

Resilienzforschung wird die traditionelle klinische Forschung ergänzen, Strategien fördern, die Gesundheit und Gesunderhaltung in den Vordergrund rücken und die Entwicklung neuer und effektiverer Präventions- und Interventionsstrategien inspirieren [12, 21]. Erkenntnisse aus Netzwerkanalysen ermöglichen die gezielte Intervention an einzelnen besonders stark vernetzten Symptomen und die Förderung von Resilienzmechanismen und -prozessen, die effektiv das gesamte Netzwerk beeinflussen [17].

Interessenkonflikt

Erklärung zu finanziellen Interessen

Forschungsförderung erhalten: nein; Honorar/geldwerten Vorteil für Referententätigkeit erhalten: nein; Bezahlter Berater/interner Schulungsreferent/Gehaltsempfänger: nein; Patent/Geschäftsanteile/Aktien (Autor/Partner, Ehepartner, Kinder) an Firma (Nicht-Sponsor der Veranstaltung): nein; Patent/Geschäftsanteile/Aktien (Autor/Partner, Ehepartner, Kinder) an Firma (Sponsor der Veranstaltung): nein.

Erklärung zu nicht finanziellen Interessen

Die Autorinnen geben an, dass kein Interessenkonflikt besteht.

\section{Danksagung}

Vielen Dank an Lisa M. Hempel. 


\section{Korrespondenzadresse}

Prof. Dr. med. Andrea Pfennig

Klinik für Psychiatrie und Psychotherapie

Fetscherstr. 74, 01307 Dresden, Deutschland

andrea.pfennig@uniklinikum-dresden.de

Literatur

[1] Werner EE, French FE, Bierman JM. The children of Kauai. A longitudinal study from the prenatal period to age ten. Honolulu: University of Hawaii Press; 1971

[2] Bonanno GA, Diminich ED. Annual Research Review: Positive adjustment to adversity - trajectories of minimal-impact resilience and emergent resilience. J Child Psychol Psychiatry 2013; 54: 378-401. doi:10.1111/jcpp.12021

[3] Masten AS. Resilienz: Modelle, Fakten \& Neurobiologie. Das ganz normale Wunder entschlüsselt. Paderborn: Junfermann; 2016

[4] Windle G. What is resilience? A review and concept analysis. Rev Clin Gerontol 2011; 21: 152-169. doi:10.1017/ S0959259810000420

[5] Antonovsky A. Health, stress and coping. The Jossey-Bass social and behavioral science series. San Francisco: JosseyBass; 1979

[6] Rutter M. Annual Research Review: Resilience - clinical implications. J Child Psychol Psychiatry 2013; 54: 474-487. doi:10.1111/j.1469-7610.2012.02615.x

[7] Norris FH, Tracy M, Galea S. Looking for resilience: understanding the longitudinal trajectories of responses to stress. Soc Sci Med 2009; 68: 2190-2198. doi:10.1016/j. socscimed.2009.03.043

[8] Bonanno GA, Westphal M, Mancini AD. Resilience to loss and potential trauma. Annu Rev Clin Psychol 2011; 7: 511-535. doi:10.1146/annurev-clinpsy-032210-104526

[9] Luthar SS, Cicchetti D, Becker B. The construct of resilience: a critical evaluation and guidelines for future work. Child Dev 2000; 71: 543-562. doi:10.1111/1467-8624.00164

[10] Deegan PE. The importance of personal medicine: a qualitative study of resilience in people with psychiatric disabilities. Scand J Public Health Suppl 2005; 66: 29-35. doi:10.1080/14034950510033345

[11] Collishaw S, Pickles A, Messer J et al. Resilience to adult psychopathology following childhood maltreatment: Evidence from a community sample. Child Abuse Negl 2007; 31: 211-229. doi:10.1016/j.chiabu.2007.02.004
[12] Kalisch R, Baker DG, Basten U et al. The resilience framework as a strategy to combat stress-related disorders. Nat Hum Behav 2017; 1: 784-790. doi:10.1038/s41562-017-0200-8

[13] Zautra AJ, Hall JS, Murray KE. Resilience: A new Definition of Health for People and Communities. In: Reich JW, Zautra A, Hall JS, Hrsg. Handbook of adult resilience. New York, London: Guilford; 2012: 3-34

[14] Haarig F, Berndt C, Kühnert M et al. Was ist Betroffenen wichtig? Bestimmung patientennaher Therapiezieldimensionen in der Behandlung von bipolaren Störungen. Zeitschrift für Psychiatrie, Psychologie und Psychotherapie 2016; 64: 111-120. doi:10.1024/1661-4747/a000269

[15] Windle G, Bennett KM, Noyes J. A methodological review of resilience measurement scales. Health Qual Life Outcomes 2011; 9: 8. doi:10.1186/1477-7525-9-8

[16] Borsboom D. A network theory of mental disorders. World Psychiatry 2017; 16: 5-13. doi:10.1002/wps.20375

[17] McNally RJ. Can network analysis transform psychopathology? Behav Res Ther 2016; 86: 95-104. doi:10.1016/j. brat.2016.06.006

[18] Kalisch R, Cramer AO], Binder $\mathrm{H}$ et al. Deconstructing and Reconstructing Resilience: A Dynamic Network Approach. Perspect Psychol Sci 2019; 14: 765-777. doi:10.1177/1745691619855637

[19] van Borkulo C, Boschloo L, Borsboom D et al. Association of Symptom Network Structure With the Course of corrected Depression. JAMA Psychiatry 2015; 72: 1219-1226. doi:10.1001/jamapsychiatry.2015.2079

[20] Masten AS. Ordinary magic: Resilience processes in development. American Psychologist 2001; 56: 227-238. doi:10.1037//0003-066X.56.3.227

[21] Sapienza JK, Masten AS. Understanding and promoting resilience in children and youth. Curr Opin Psychiatry 2011; 24: 267-273. doi:10.1097/YCO.0b013e32834776a8

\section{Bibliografie}

Nervenheilkunde 2021; 40: 242-246

DOI 10.1055/a-1298-0656

ISSN $0722-1541$

(c) 2021. Thieme. All rights reserved. Georg Thieme Verlag KG, Rüdigerstraße 14, 70469 Stuttgart, Germany 


\section{Punkte sammeln auf CME.thieme.de}

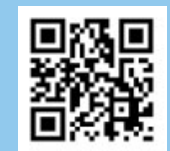

Diese Fortbildungseinheit ist bis zu 12 Monate online für die Teilnahme verfügbar.

Den genauen Einsendeschluss finden Sie beim Modul auf https://cme.thieme.de/CXGZBZ4.

Sollten Sie Fragen zur Online-Teilnahme haben, finden Sie unter https://cme.thieme.de/hilfe

eine ausführliche Anleitung. Wir wünschen viel Erfolg beim Beantworten

der Fragen!

Unter https://eref.thieme.de/CXGZBZ4 oder über den QR-Code kommen Sie

direkt zum Artikel.

VNR 2760512021160213652

\section{Frage 1}

Welche Aussage spiegelt das derzeitige Verständnis von Resilienz am treffendsten wider?

A Resilienz ist das Gegenteil von Risiko.

B Resilienz beschreibt die einzigartige Unverwundbarkeit einzelner Individuen.

C Resilienz sagt vorher, wer angesichts eines Stressors gesund bleibt und wer krank wird.

D Resilienz ist ein aktiver, dynamischer Prozess der effektiven Anpassung an einen Stressor.

E Resilienz beschreibt überdauernde Persönlichkeitseigenschaften, die Gesundheit fördern.

\section{Frage 2}

Das Phänomen Resilienz wurde vor mindestens 60 Jahren erstmals bewusst beobachtet in Untersuchungen von...
A Soldaten im Auslandseinsatz.
B Kindern und Jugendlichen in widrigen Lebensumständen.
C Börsenhändlern in Finanzkrisen.
D kürzlich verwitweten Ehepartnern.
$E$ in Klöstern lebenden Nonnen.

\section{Frage 3}

Die Zusammenfassung und Vergleichbarkeit existierender Ergebnisse der Resilienzforschung ist deutlich erschwert. Wieso?

A Die Anzahl der Studienergebnisse ist so umfangreich, dass niemand einen Überblick gewinnen kann.

B Es existieren zahlreiche verschiedene Ansätze zur Definition, Operationalisierung und Messung von Resilienz.

C Resilienz hat in jeder Sprache eine leicht andere Bedeutung.

D Der Resilienzbegriff ist erst wenige Jahre alt und daher noch unklar umrissen.

E Resilienz ist ein so seltenes Phänomen, dass kaum Beobachtungen vorliegen.

\section{Frage 4}

Was ist keine typische Charakteristik einer resilienten Entwicklung?

A Der Verlauf wird durch genetische, biologische, psychologische, interpersonelle und umgebungsbedingte Variablen beeinflusst.

B Resiliente Entwicklungen sind individuell unterschiedlich und durch verschiedene Wirkprozesse bedingt.

C In Reaktion auf einen Stressor treten milde Symptome und Funktionseinschränkungen auf.

D Symptome und Funktionsfähigkeit variieren unabhängig von auftretenden Stressoren.

E Funktionseinschränkungen sind vorübergehend.

\section{Frage 5}

Was haben resiliente Anpassungsprofile gemeinsam?

A Einer graduellen Zunahme von Symptomen folgt eine plötzliche Abnahme von Symptomen.

B Beschwerden treten zeitverzögert vom Stressor auf.

C Sie führen im Verlauf zu einem Zustand psychischer Gesundheit und Funktionsfähigkeit.

D Das Auftreten eines Stressors verursacht keine Veränderung im Funktionsniveau.

E Die Emotionsregulationsfähigkeit ist besonders niedrig ausgeprägt.

\section{Frage 6}

Wie unterscheiden sich Resistenz und Resilienz im engeren Sinne?

A Resistenz und Resilienz unterscheiden sich vorrangig im Ausmaß der Stressorbelastung.

B Resistenz und Resilienz unterscheiden sich im Ausmaß der beobachtbaren Reaktion auf den Stressor.

C Resistenz und Resilienz unterscheiden sich hinsichtlich der Art des Stressors.

D Resistenz und Resilienz sind Synonyme.

E Resistenz und Resilienz sind konzeptuell identisch und unterscheiden sich im angewandten Messverfahren. 


\section{Punkte sammeln auf CME.thieme.de}

Fortsetzung ...

\section{Frage 7}

Das binäres Denkmuster krank-versus-gesund bringt in der Resilienzforschung folgende Nachteile mit sich. Welche Aussage gehört nicht dazu?

A Resilienz und Risiko werden als gegensätzliche Pole eines Kontinuums interpretiert.

B Der Informationszugewinn zur Risikoforschung ist gering.

C Symptomausprägungen werden durch diskrete, kontinuierliche Werte abgebildet.

D Faktoren, die Gesundheit vorhersagen, sagen auch Krankheit vorher.

E Ressourcen werden als Gegenteil von Risikofaktoren verstanden.

\section{Frage 8}

Welche Aussage zu Resilienzfaktoren trifft nicht zu?

A Resilienzfaktoren sagen eine resiliente Entwicklung vorher.

B Resilienzfaktoren werden auf individueller, sozialer und gesellschaftlicher Ebene untersucht.

C Resilienzfaktoren sind immer feststehende, unveränderliche Charakteristika einer Person.

D Resilienzfaktoren wirken durch die Aktivierung von Resilienzmechanismen.

E Resilienfaktoren können als zusätzliche Knotenpunkte in Netzwerkanalysen integriert werden.

\section{Frage 9}

Das einfachste Studiendesign zur Untersuchung von Resilienz erfasst u. a....

A die Ausprägung einer Ergebnisvariable nach der Stressorexposition.

B die Ausprägung einer Ergebnisvariable während der Stressorexposition.

C die Ausprägung einer Ergebnisvariable vor der Stressorexposition.

D die Ausprägung einer Ergebnisvariable vor und nach der Stressorexposition.

E die Ausprägung einer Ergebnisvariable 3 Monate nach Stressorexposition.

\section{Frage 10}

Die Symptomenkonstellationen psychischer Störungen können durch Netzwerke modelliert werden. Durch welche Eigenschaften zeichnen sich resiliente Netzwerke aus?

A Geringer Aktivierungsgrad und/oder geringe Konnektivität der Symptome.

B Hoher Aktivierungsgrad und hohe Konnektivität der Symptome.

C Sogenannte „tipping points“ werden besonders schnell überschritten.

D Externe Stressoren aktivieren alle Knotenpunkte gleichzeitig.

E Die Aktivierungsstärke dieser Netzwerke wird einzig durch biologische Mechanismen realisiert. 